\title{
Time-Frequency Analysis of EEG Asymmetry Using Bivariate Empirical Mode Decomposition
}

\author{
Cheolsoo Park, David Looney, Preben Kidmose, Michael Ungstrup, and Danilo P. Mandic, Senior Member, IEEE
}

\begin{abstract}
A novel method is introduced to determine asymmetry, the lateralization of brain activity, using extension of the algorithm empirical mode decomposition (EMD). The localized and adaptive nature of EMD make it highly suitable for estimating amplitude information across frequency for nonlinear and nonstationary data. Analysis illustrates how bivariate extension of EMD (BEMD) facilitates enhanced spectrum estimation for multichannel recordings that contain similar signal components, a realistic assumption in electroencephalography (EEG). It is shown how this property can be used to obtain a more accurate estimate of the marginalized spectrum, critical for the localized calculation of amplitude asymmetry in frequency. Simulations on synthetic data sets and feature estimation for a brain-computer interface (BCI) application are used to validate the proposed asymmetry estimation methodology.
\end{abstract}

Index Terms-Asymmetry ratio, bivariate empirical mode decomposition (BEMD), cognitive task, electroencephalography (EEG), empirical mode decomposition (EMD).

\section{INTRODUCTION}

B RAIN-COMPUTER interface (BCI) is a novel research paradigm that facilitates computer-aided control using brain activity, and has found application across bioengineering fields, such as neuroprosthetics. Due to its noninvasive nature and affordable recording equipment, the electroencephalography (EEG) is the most convenient means to measure neurophysiological activity in real-time BCI systems [1]. The level of asymmetry observed in EEG, that is, the lateralization of activity between left and right brain hemispheres, has been found to be of much interest in the detection and estimation of brain electrical activity corresponding to cognitive processes [2], [3].

Asymmetry studies have been addressed both the full EEG spectrum [4]-[6] and also specific frequency bands of interest [7]-[9]. For instance, recent work focused on detecting asymmetry in the alpha band [5], [7], as the suppression of EEG activity in low-frequency bands is associated with cognitive engagement. McKee et al. [8] found that linguistic tasks, in comparison to music tasks, were characterized by greater alpha suppression. It was established in [9] that the lateral asymmetries in contingent negative variation were caused by cognitive ac-

Manuscript received June 06, 2010; revised November 16, 2010; accepted January 20, 2011. Date of publication February 22, 2011; date of current version August 10, 2011

C. Park, D. Looney, and D. P. Mandic are with the Department of Electrical and Electronic Engineering, Imperial College London, SW7 2BT London, U.K.

P. Kidmose is with the Department of Medical Technology, Engineering College of Aarhus, 8000 Aarhus, Denmark.

M. Ungstrup is with the Widex Widex A/S, 3540 Lynge, Denmark.

Digital Object Identifier 10.1109/TNSRE.2011.2116805 tivity of brain. Davidson found that verbal and spatial tasks were characterized by different levels of EEG asymmetry in multiple frequency bands [5]. Furthermore, neuropsychological tasks "Verbal Fluency," "the Tower of London," and "Corsi's Recurring Blocks" produced specific asymmetry patterns in the delta and theta bands [6].

Although these results illustrate that asymmetry is a robust measure for classifying different mental tasks [10], [11], existing studies have employed standard signal processing techniques based on Fourier analysis [1], [11]-[13] and thus inherit the well-known problems associated with standard spectrum estimation. In addition, standard techniques project the data onto linear orthogonal basis functions, and are thus suboptimal for processing of nonlinear and nonstationary real world data (such as EEG).

In this work, we propose to estimate asymmetry using empirical mode decomposition (EMD) [14], a fully data-driven technique for decomposing the signal into AM/FM components which reflect its natural oscillations. EMD makes no prior assumptions on the data and, as such, it is suitable for the analysis of nonlinear and nonstationary processes, and has been successfully employed in the analysis of EEG [15], [16]. Using EMD, frequency and amplitude information can be analyzed locally, providing a highly accurate insight into signal dynamics, making it an ideal candidate for estimating asymmetry. The asymmetry estimation paradigm is compared for both the real-valued and complex-valued EMD algorithm, showing that bivariate EMD (BEMD) produces enhanced spectrum estimation for multichannel recordings that contain similar oscillating components [17], a realistic assumption in EEG. This, in turn, yields a more accurate estimate of the marginalized spectra, critical issue in the localized calculation of amplitude asymmetry in frequency. Simulations on both synthetic signals and real world cognitive EEG tasks illustrate the usefulness of the proposed approach for calculating asymmetry.

\section{EMPIRICAL MODE DECOMPOSITION ALGORITHM}

\section{A. Empirical Mode Decomposition}

Huang et al. [14] developed EMD to perform a highly localized time-frequency estimation in a data-driven fashion. This is achieved by decomposing the signal into a finite set of AM/FM components, called "intrinsic mode functions" (IMFs). An IMF is a function for which the number of extrema and the number of zero crossings differ at most by one, and the mean of the two envelopes associated with the local maxima and local minima is approximately zero. The IMF can thus be regarded as a monocomponent signal. The details of the EMD operation are outlined in algorithm 1 [14]. The first IMF is subtracted from the 
original data, $r(t)=x(t)-d(t)$, and the procedure is applied iteratively to the residue, $r(t)$, until it becomes constant or contains no more oscillations. The stopping criterion uses the mode amplitude

\section{Algorithm 1. The standard EMD algorithm}

1) Let $\tilde{x}(t)=x(t)(x(t)$ is original signal).

2) Identify all local maxima and minima of $\tilde{x}(t)$.

3) Find a lower "envelope," $e_{l}(t)$ that interpolates all local minima.

4) Find an upper "envelope," $e_{u}(t)$ that interpolates all local maxima.

5) Calculate the local mean value, $m(t)=$ $\left(e_{l}(t)+e_{u}(t)\right) / 2$.

6) Subtract the local mean value from $\tilde{x}(t)$, $d(t)=\tilde{x}(t)-m(t)$.

7) Let $\tilde{x}(t)=d(t)$ and go to step 2); repeat until $d(t)$ becomes an IMF.

$a(t):=\left(e_{u}(t)-e_{l}(t)\right) / 2$ and the evaluation function $\sigma(t):=$ $|m(t) / a(t)|$, so that sifting is iterated until $\sigma(t)<\theta_{1}$ for some prescribed fraction $(1-\alpha)$ of the total duration, while $\sigma(t)<\theta_{2}$ for the remaining fraction [18]. The default value of $\left[\theta_{1}, \theta_{2}, \alpha\right]$ is $[0.05,0.5,0.05]$. The signal $x(t)$ decomposed by the EMD algorithm can thus be written as

$$
x(t)=\sum_{i=1}^{M} c_{i}(t)+r(t)
$$

$c_{i}(t), i=1, \ldots, M$, is the set of IMFs. Due to their monocomponent nature, it is convenient to apply the Hilbert transform to the IMFs to obtain a time-frequency representation. The Hilbert transform of an IMF $c_{i}(t)$ is given by

$$
H\left[c_{i}(t)\right]=\frac{1}{\pi} P \int_{-\infty}^{\infty} \frac{c_{i}\left(t^{\prime}\right)}{t-t^{\prime}} d t^{\prime}
$$

where symbol $P$ indicates the Cauchy principal value. The analytic signal is then obtained as

$$
X(t)=\sum_{i=1}^{M}\left(c_{i}(t)+j H\left[c_{i}(t)\right]\right)=\sum_{i=1}^{M} a_{i}(t) \cdot e^{j \theta_{i}(t)}
$$

and is described by its amplitude and phase functions, $a_{i}(t)$ and $\theta_{i}(t)$. The instantaneous frequency is calculated as the derivative of the phase function, $w_{i}(t)=\left(d \theta_{i}\right) /(d t)$ [19]; a plot of the amplitude $a_{i}(t)$ versus time $t$ and instantaneous frequency $w_{i}(t)$, that is, amplitude contours on the time-frequency plane is called the Hilbert-Huang spectrum (HHS), $\mathbf{H}(w, t)$, a three-dimensional time-frequency representation of a signal. The marginal Hilbert spectrum (MHS), $h(w)$, is calculated by marginalising the amplitude of the HHS over time

$$
h(w)=\int_{0}^{T} \mathbf{H}(w, t) d t
$$

where $T$ is the total data length. The advantage of MHS for EEG applications has been addressed in [20]-[22].

\section{B. Complex Extension of EMD}

To obtain a set of $\mathrm{M}$ complex/bivariate IMFs, $\gamma_{i}(t), \mathrm{i}=$ $1, \ldots, \mathrm{M}$, from a complex signal $z(t)$, the following procedure, algorithm 2, is adopted (bivariate EMD) [23].

Algorithm 2. The bivariate EMD algorithm

1) Let $\tilde{z}(t)=z(t)$;

2) To obtain $K$ signal projections, given by $\left\{p_{\theta_{k}}\right\}_{k=1}^{K}$, project the complex signal $\tilde{z}(t)$, by using a unit complex number $e^{-j \theta_{k}}$, in the direction of $\theta_{k}$, as

$$
p_{\theta_{k}}(t)=\Re\left(e^{-j \theta_{k}} \tilde{z}(t)\right), \quad k=1, \ldots, K
$$

where $\Re(\cdot)$ denotes the real part of a complex number, and $\theta_{k}=2 k \pi / K$;

3) Find the locations $\left\{t_{j}^{k}\right\}_{k=1}^{K}$ corresponding to the maxima of $\left\{p_{\theta_{k}}(t)\right\}_{k=1}^{K}$;

4) Interpolate (using spline interpolation) between the maxima points $\left[t_{j}^{k}, \tilde{z}\left(t_{j}^{k}\right)\right]$, to obtain the envelope curves $\left\{e_{\theta_{k}}(t)\right\}_{k=1}^{K}$;

5 ) Obtain the arithmetic mean of all the envelope curves, $m(t)$, and subtract from the input signal, that is, $d(t)=\tilde{z}(t)-m(t)$. Let $\tilde{z}(t)=d(t)$ and go to step 2)

6) Repeat until $d(t)$ becomes an IMF;

Similarly to real-valued EMD, once the first IMF, $\gamma_{1}(t)$, is obtained the procedure is applied iteratively to the residual $r(t)=$ $z(t)-d(t)$ to extract all the complex IMFs, wihch rotate around zero [23]. In our simulations, the sifting process was stopped once the magnitude of $d(t)$ satisfied the real-valued stopping criterion described in [18] and the number of projections for all BEMD decomposition, $K$, was 16.

Earlier results [17] illustrate that in applications involving a pair of real valued sources, $x_{1}$ and $x_{2}$, it is advantageous to apply BEMD to the complex signal $z=x_{1}+j x_{2}$. The real and imaginary components of the decomposition can then be viewed as two separate sets of IMFs, corresponding respectively to the real and imaginary components of the input. The advantage of applying this bivariate approach, compared to two individual real valued EMD operations, is that by design it improves the stability and locality of each set of IMFs.

The BEMD operation uses multiple projections of the complex signal; each projection is real-valued and is used to describe the amplitude/envelope of the signal in a given direction. It is important to note that each projection is a function of both the real and imaginary parts and will therefore yield improved instantaneous amplitude estimation if at a given scale the real and imaginary parts share the same oscillatory modes. This is illustrated in more detail in the next section.

\section{Performance Comparison of EMD and BEMD}

The following two sections investigate the capacity of BEMD to achieve a more robust estimate of spectrum information compared to EMD, as accurate estimation of power spectrum is crucially important for asymmetry analysis. This is achieved by comparing their performance at 1) the IMF level and 2) the level of marginal Hilbert spectrum. 
TABLE I

SINUSOID RECONSTRUCTION ( $\hat{s}_{\text {EMD AND }} \hat{s}_{\text {BEMD }}$ ) RESULTS IN SNR FOR DIFFERENT FREQUENCIES AND INITIAL NOISE LEVELS

\begin{tabular}{c|c|c|c|c|c|c}
\hline Algorithm & Freq. & $2 \mathrm{~Hz}$ & $6 \mathrm{~Hz}$ & $12 \mathrm{~Hz}$ & $25 \mathrm{~Hz}$ & $44 \mathrm{~Hz}$ \\
\hline EMD & $5 \mathrm{~dB}$ & $17.2 \mathrm{~dB}$ & $13.3 \mathrm{~dB}$ & $11.1 \mathrm{~dB}$ & $9.0 \mathrm{~dB}$ & $6.5 \mathrm{~dB}$ \\
BEMD & $5 \mathrm{~dB}$ & $18.8 \mathrm{~dB}$ & $13.8 \mathrm{~dB}$ & $11.7 \mathrm{~dB}$ & $9.5 \mathrm{~dB}$ & $7.4 \mathrm{~dB}$ \\
\hline EMD & $0 \mathrm{~dB}$ & $12.6 \mathrm{~dB}$ & $9.7 \mathrm{~dB}$ & $7.5 \mathrm{~dB}$ & $5.5 \mathrm{~dB}$ & $4.1 \mathrm{~dB}$ \\
BEMD & $0 \mathrm{~dB}$ & $14.2 \mathrm{~dB}$ & $10.9 \mathrm{~dB}$ & $9.0 \mathrm{~dB}$ & $6.8 \mathrm{~dB}$ & $5.6 \mathrm{~dB}$ \\
\hline EMD & $-5 \mathrm{~dB}$ & $9.2 \mathrm{~dB}$ & $6.0 \mathrm{~dB}$ & $4.3 \mathrm{~dB}$ & $3.0 \mathrm{~dB}$ & $2.2 \mathrm{~dB}$ \\
BEMD & $-5 \mathrm{~dB}$ & $10.7 \mathrm{~dB}$ & $7.7 \mathrm{~dB}$ & $5.9 \mathrm{~dB}$ & $4.4 \mathrm{~dB}$ & $3.0 \mathrm{~dB}$ \\
\hline EMD & $-10 \mathrm{~dB}$ & $6.3 \mathrm{~dB}$ & $3.5 \mathrm{~dB}$ & $2.3 \mathrm{~dB}$ & $1.3 \mathrm{~dB}$ & $0.9 \mathrm{~dB}$ \\
BEMD & $-10 \mathrm{~dB}$ & $7.6 \mathrm{~dB}$ & $4.2 \mathrm{~dB}$ & $3.0 \mathrm{~dB}$ & $2.1 \mathrm{~dB}$ & $1.3 \mathrm{~dB}$ \\
\hline
\end{tabular}

1) IMF Estimation: The aim of asymmetry estimation is to characterise the degree of similarity between two data sources across frequency. In scenarios where the sources share similar components, we shall illustrate, following our previous work in [17], that the BEMD gives advantage by simultaneously modelling joint oscillating modes at each IMF level.

In this work, we decompose the signal $x=s_{f}+v$ using EMD, where $s_{f}$ is a sinusoid of frequency $f$ and $v$ is a realisation of white Gaussian noise (WGN), and subsequently apply the Wiener filter. ${ }^{1}$ to the IMFs to obtain an estimate of the sinusoid, $\hat{s}_{\text {EMD }}$ The more accurate the estimate of $s_{f}$, the more accurately the IMFs represent the original input components. Additionally, BEMD was performed on $z=\left(s_{f}+v_{r}\right)+j\left(s_{f}+v_{i}\right)$ where $v_{r}$ and $v_{i}$ denote different realisations of WGN in both the real and imaginary parts of $z$. For comparison with the EMD operation, the Wiener filter was applied to the real part only of the bivariate IMFs to obtain an estimate for the sinusoid,

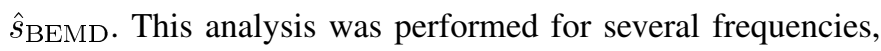
and over four signal-to-noise ratio $\left(\mathrm{SNR}_{\text {init. }}\right)$ levels. The analysis was also extended to $x=f_{A}\left(s_{f}\right)+v$ in the case of EMD and $z=f_{A}\left(s_{f}\right)+v_{r}+j\left(f_{A}\left(s_{f}\right)+v_{i}\right)$ in the case of BEMD where $f_{A}(\cdot)$ denotes an amplitude modulation operation (using 1 or $2 \mathrm{~Hz}$ sinusoid) to illustrate component estimation for signals with changing amplitudes. The sampling frequency was $256 \mathrm{~Hz}$ and the signal length $10 \mathrm{~s}$.

The average SNR of the reconstructed uniform amplitude sinusoids over 50 simulations using EMD and BEMD are given in Table I. The superior performance of BEMD is evident for all considered simulations. Component estimation results for sinusoids modulated at 1 and $2 \mathrm{~Hz}$ are shown respectively in Tables II and III. The BEMD algorithm consistently allowed for better component estimation.

For rigour, the simulations were performed over a range of parameters for the stopping criterion, as BEMD and EMD often require different numbers of sifting operations even when using the same stopping criterion. Component estimation performance for the signal $s_{f}$, for $f=12 \mathrm{~Hz}$ and $f=44 \mathrm{~Hz}$ and for $0 \mathrm{~dB}$ and $-10 \mathrm{~dB}$ SNR, for different numbers of sifting operations (by adjusting the stopping criterion) are shown in Fig. 1, where, as before, BEMD consistently outperform EMD. These results illustrate that the enhanced BEMD performance is caused by more accurate component estimation and not by virtue of better sifting.
TABLE II

ReCONSTRUCtion Results of a 1 Hz AMPlitude Modulated SINUSOID ( $\hat{s}_{\text {EMD }}$ AND $\hat{s}_{\text {BEMD }}$ ) IN SNR FOR DIFFERENT FREQUENCIES AND INITIAL NOISE LEVELS

\begin{tabular}{c|c|c|c|c|c|c}
\hline Algorithm & FNeq. & $2 \mathrm{~Hz}$ & $6 \mathrm{~Hz}$ & $12 \mathrm{~Hz}$ & $25 \mathrm{~Hz}$ & $44 \mathrm{~Hz}$ \\
\hline EMD & $5 \mathrm{~dB}$ & $17.1 \mathrm{~dB}$ & $12.6 \mathrm{~dB}$ & $10.6 \mathrm{~dB}$ & $8.8 \mathrm{~dB}$ & $6.5 \mathrm{~dB}$ \\
BEMD & $5 \mathrm{~dB}$ & $18.1 \mathrm{~dB}$ & $13.8 \mathrm{~dB}$ & $11.6 \mathrm{~dB}$ & $9.5 \mathrm{~dB}$ & $6.9 \mathrm{~dB}$ \\
\hline EMD & $0 \mathrm{~dB}$ & $12.7 \mathrm{~dB}$ & $9.5 \mathrm{~dB}$ & $7.5 \mathrm{~dB}$ & $5.4 \mathrm{~dB}$ & $3.8 \mathrm{~dB}$ \\
BEMD & $0 \mathrm{~dB}$ & $14.1 \mathrm{~dB}$ & $10.3 \mathrm{~dB}$ & $8.1 \mathrm{~dB}$ & $6.1 \mathrm{~dB}$ & $5.1 \mathrm{~dB}$ \\
\hline EMD & $-5 \mathrm{~dB}$ & $9.0 \mathrm{~dB}$ & $5.8 \mathrm{~dB}$ & $4.2 \mathrm{~dB}$ & $2.8 \mathrm{~dB}$ & $2.1 \mathrm{~dB}$ \\
BEMD & $-5 \mathrm{~dB}$ & $10.0 \mathrm{~dB}$ & $7.5 \mathrm{~dB}$ & $5.9 \mathrm{~dB}$ & $4.3 \mathrm{~dB}$ & $3.0 \mathrm{~dB}$ \\
\hline EMD & $-10 \mathrm{~dB}$ & $5.8 \mathrm{~dB}$ & $3.3 \mathrm{~dB}$ & $2.2 \mathrm{~dB}$ & $1.3 \mathrm{~dB}$ & $0.9 \mathrm{~dB}$ \\
BEMD & $-10 \mathrm{~dB}$ & $7.0 \mathrm{~dB}$ & $4.3 \mathrm{~dB}$ & $3.0 \mathrm{~dB}$ & $2.1 \mathrm{~dB}$ & $1.3 \mathrm{~dB}$ \\
\hline
\end{tabular}

TABLE III

ReConstruction Results of 2 Hz Amplitude Modulated SINUSOID ( $\hat{s}_{\text {EMD }}$ AND $\hat{s}_{\text {BEMD }}$ ) IN SNR FOR DIFFERENT FREQUENCIES AND INITIAL NOISE LEVELS

\begin{tabular}{c|c|r|r|c|c|c}
\hline Algorithm & Freq. & $2 \mathrm{~Hz}$ & $6 \mathrm{~Hz}$ & $12 \mathrm{~Hz}$ & $25 \mathrm{~Hz}$ & $44 \mathrm{~Hz}$ \\
\hline EMD & $5 \mathrm{~dB}$ & $16.8 \mathrm{~dB}$ & $13.0 \mathrm{~dB}$ & $11.0 \mathrm{~dB}$ & $8.7 \mathrm{~dB}$ & $6.5 \mathrm{~dB}$ \\
BEMD & $5 \mathrm{~dB}$ & $18.0 \mathrm{~dB}$ & $13.9 \mathrm{~dB}$ & $11.7 \mathrm{~dB}$ & $9.5 \mathrm{~dB}$ & $6.9 \mathrm{~dB}$ \\
\hline EMD & $0 \mathrm{~dB}$ & $12.3 \mathrm{~dB}$ & $9.6 \mathrm{~dB}$ & $7.4 \mathrm{~dB}$ & $5.4 \mathrm{~dB}$ & $3.8 \mathrm{~dB}$ \\
BEMD & $0 \mathrm{~dB}$ & $13.9 \mathrm{~dB}$ & $10.4 \mathrm{~dB}$ & $8.4 \mathrm{~dB}$ & $6.2 \mathrm{~dB}$ & $5.1 \mathrm{~dB}$ \\
\hline EMD & $-5 \mathrm{~dB}$ & $9.0 \mathrm{~dB}$ & $6.0 \mathrm{~dB}$ & $4.4 \mathrm{~dB}$ & $2.8 \mathrm{~dB}$ & $2.1 \mathrm{~dB}$ \\
BEMD & $-5 \mathrm{~dB}$ & $10.4 \mathrm{~dB}$ & $7.5 \mathrm{~dB}$ & $5.9 \mathrm{~dB}$ & $4.2 \mathrm{~dB}$ & $3.0 \mathrm{~dB}$ \\
\hline EMD & $-10 \mathrm{~dB}$ & $5.9 \mathrm{~dB}$ & $3.3 \mathrm{~dB}$ & $2.2 \mathrm{~dB}$ & $1.3 \mathrm{~dB}$ & $0.9 \mathrm{~dB}$ \\
BEMD & $-10 \mathrm{~dB}$ & $7.0 \mathrm{~dB}$ & $4.1 \mathrm{~dB}$ & $3.0 \mathrm{~dB}$ & $2.1 \mathrm{~dB}$ & $1.3 \mathrm{~dB}$ \\
\hline
\end{tabular}

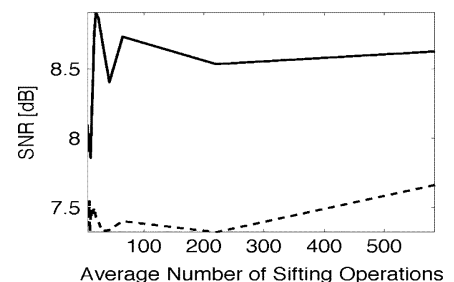

(a)

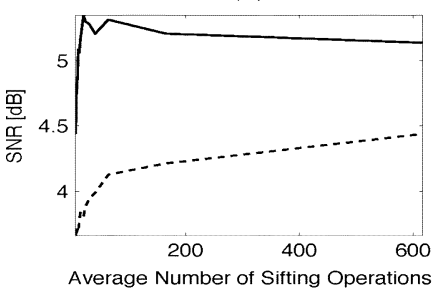

(c)

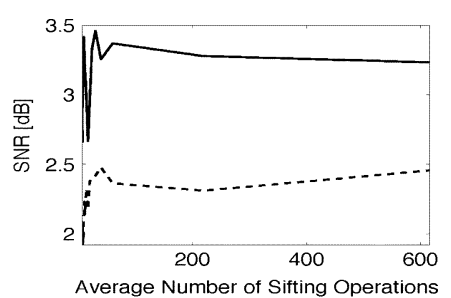

(b)

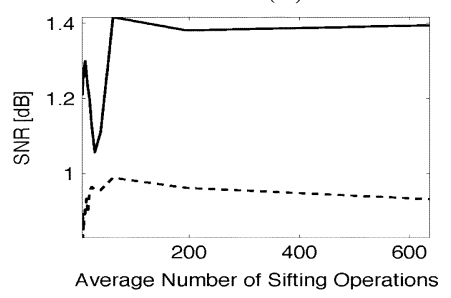

(d)
Fig. 1. Reconstruction results of $1 \mathrm{~Hz}$ amplitude modulated sinusoid for different frequencies, average number of sifting operations and SNR (" $--\ldots$ " EMD, "-" BEMD). (a) $f=12 \mathrm{~Hz}$, SNR $=0 \mathrm{~dB}$, (b) $f=12 \mathrm{~Hz}$, SNR $=$ $-10 \mathrm{~dB}$, (c) $f=44 \mathrm{~Hz}, \mathrm{SNR}=0 \mathrm{~dB}$, (d) $f=44 \mathrm{~Hz}$, SNR $=-10 \mathrm{~dB}$.

It can therefore be deduced that mutual information between the real and the imaginary parts of the BEMD allowed for a more accurate estimate of the common components at the IMF level. This is intuitive, as the performance of any EMD algorithm is dependent on accurate local mean estimation which, in the case of BEMD, is enhanced by two observations of any shared signal elements.

2) Spectrum Estimation: We shall now illustrate that the superior performance of BEMD over EMD supports a more accurate estimate of the marginal Hilbert spectrum (MHS) compared to EMD. As before, EMD and BEMD were applied to sinusoids with added WGN. In the case of BEMD, the MHS 

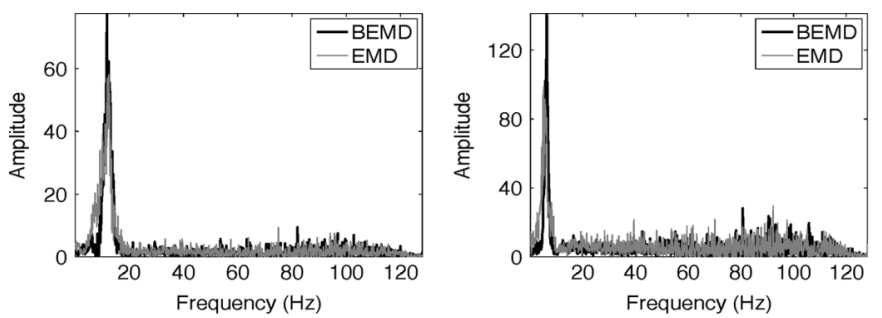

(a)

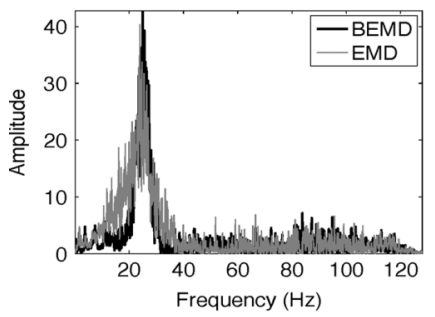

(c)

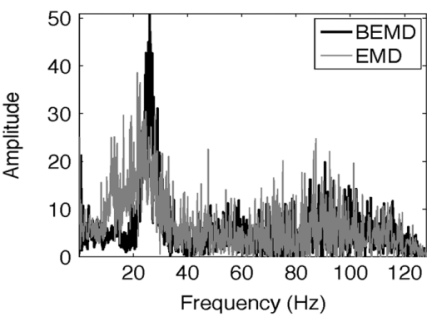

(d)

Fig. 2. The marginal Hilbert spectrum of EMD and BEMD. In all scenarios, the MHS of BEMD is more localized in frequency. (a) $6 \mathrm{~Hz}$ with $0 \mathrm{~dB}$ noise. (b) $6 \mathrm{~Hz}$ with $-5 \mathrm{~dB}$ noise. (c) $25 \mathrm{~Hz}$ with $0 \mathrm{~dB}$ noise. (d) $25 \mathrm{~Hz}$ with $-5 \mathrm{~dB}$ noise.

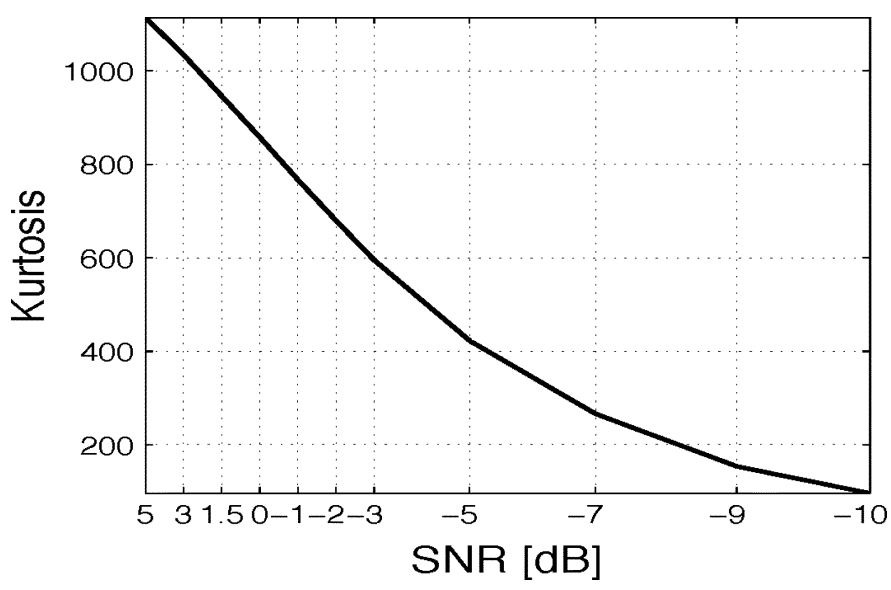

Fig. 3. The kurtosis of the periodogram based power spectrum for a sine wave increasing noise levels. Kurtosis increases for high SNR, indicating a more concentrated spectrum.

was calculated for the real part of the bivariate IMFs for $z=$ $s_{f}+v_{r}+j\left(s_{f}+v_{i}\right)$ where $s_{f}$ is a sinusoid of frequency $f$ and $v_{r}$ and $v_{i}$ are different realizations of WGN with identical statistics. In the case of EMD, the MHS was estimated for the IMFs of $s_{f}+v_{r}$. Fig. 2 compares the MHS of EMD and BEMD for several frequencies and SNRs, showing a more localized result produced by BEMD, particularly at low SNR.

To quantify the performance, the kurtosis of $x$, defined as

$$
k=\frac{E\left\{(x-\mu)^{4}\right\}}{\sigma^{4}}
$$

was used, where $\mu$ is the mean of $x, \sigma$ is the standard deviation of $x$ and $\mathrm{E}\{\cdot\}$ the expected value operates. Since kurtosis measures the peakedness of a distribution, for spectra of narrow band components localized in frequency such as a sinusoid, a high kurtosis value indicates an accurate MHS. This is illustrated in Fig. 3 for the marginalized spectrum of a single sinusoid with added WGN; the graphs were calculated for increasing levels of

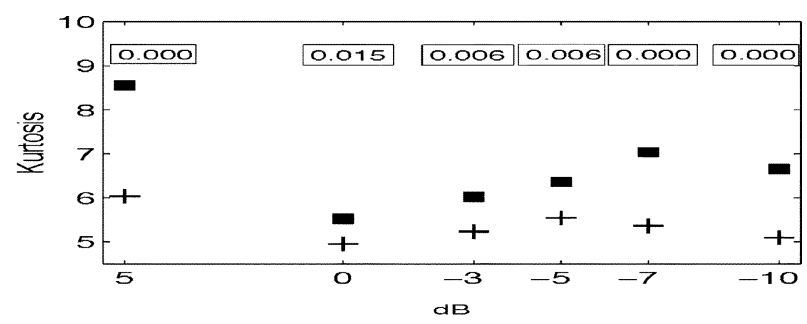

(a)

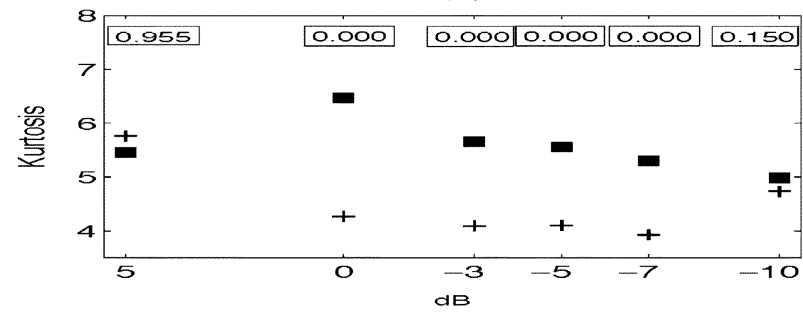

(b)

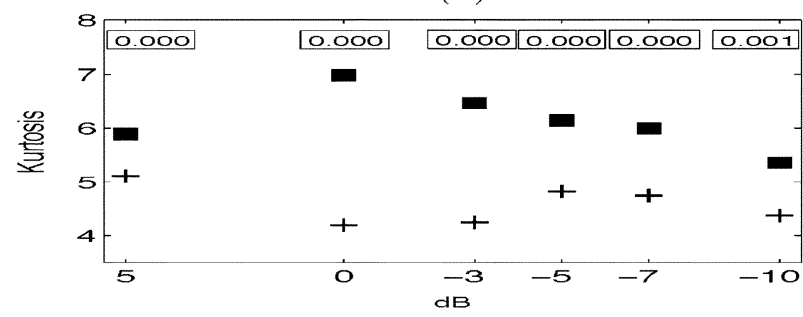

(c)

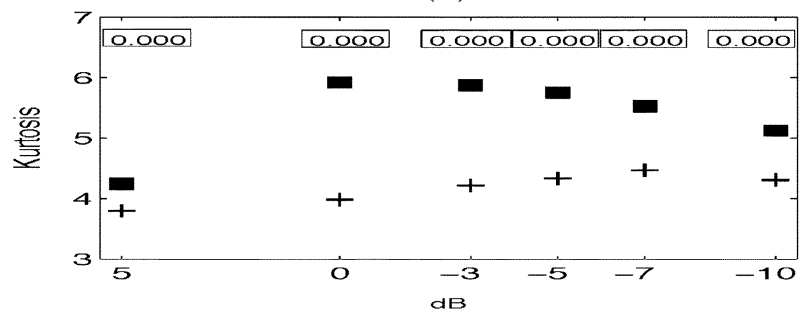

(d)

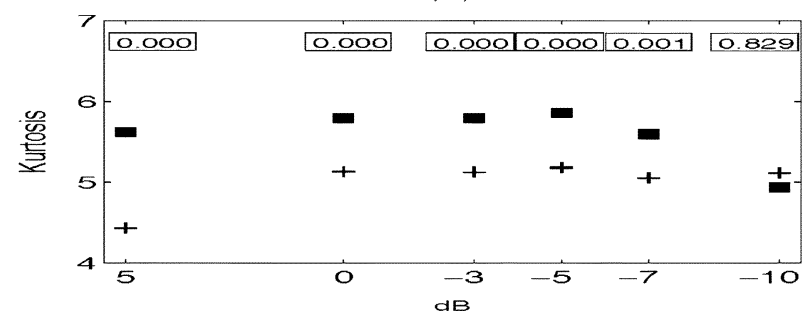

(e)

Fig. 4. The kurtosis for the MHS of the sinusoids of frequency $f$ by EMD (cross) and BEMD (black squares) for the different noise levels. Even though the kurtosis of spectrum estimates based on EMD and BEMD are similar for $-10 \mathrm{~dB}$, the results of BEMD are almost always greater than those based on EMD, implying a more accurate MHS estimate. The values in the square boxes are one-tailed p-values of the t-test for the kurtosis of EMD and BEMD results. Their overall statistical significance, as determined by p-values, was less than 0.05. (a) $f=2 \mathrm{~Hz}$, (b) $f=6 \mathrm{~Hz}$, (c) $f=12 \mathrm{~Hz}$, (d) $f=25 \mathrm{~Hz}$, (e) $f=$ $44 \mathrm{~Hz}$.

noise. ${ }^{2}$ Fig. 4 shows average results over 100 trials where kurtosis values were calculated for EMD and BEMD for different frequencies and different SNR.

${ }^{2}$ Every plot was produced as an average of 50 realizations of WGN for the different frequency sinusoids $(64,44,32,25,16,12,8,6,4,2$, and $1 \mathrm{~Hz}$, with $256 \mathrm{~Hz}$ sampling frequency), using periodogram. 
For rigour, the degree of separation between the performance of BEMD and EMD was determined using the one-tailed t-test. The p-value, for each SNR and value for frequency, is also shown in Fig. 4. In 27 of the 30 considered simulations, the performance of the BEMD MHS was significantly better than that of EMD (p-values less than 0.05).

\section{ASYMMETRY}

Asymmetry is defined as the normalised difference between brain activity from a location on the left hemisphere and its corresponding location on the right hemisphere. In practice, the absolute value of this difference is used, however this is controversial among brain researchers as it produces results that can be highly correlated. In our analysis, the absolute value of the difference was considered as results without a modulus operation tend to be highly skewed [5].

\section{A. BEMD Based Asymmetry}

For a pair of sources, $x_{1}$ and $x_{2}$, the complex IMFs are calculated for $z=x_{1}+j x_{2}$. The real and imaginary components of the decomposition are then separated giving two sets of IMFs for the two input sources. The Hilbert transform is applied to obtain the instantaneous amplitudes $a_{1, i}(t)$ and $a_{2, i}(t)$, symbols " $i$ " and "t" denote the order of IMF and the time index, and the instantaneous phases $\theta_{1, i}(t)$ and $\theta_{1, i}(t)$ using (3) for each set of IMFs. The instantaneous frequencies, $w_{1, i}(t)$ and $w_{2, i}(t)$, are computed by differentiating $\theta_{1, i}(t)$ and $\theta_{2, i}(t)$, which can be assumed to contain similar scales [17] since they are obtained from the real and imaginary components of a single complex IMF. It is therefore feasible to compare two spectral magnitudes $a_{1, i}(t)$ and $a_{2, i}(t)$, their common instantaneous frequency can be defined by the average of their respective instantaneous frequencies as

$$
w_{1,2, i}(t)=\frac{w_{1, i}(t)+w_{2, i}(t)}{2} .
$$

The corresponding marginal Hilbert spectra $h_{1}(w)$ and $h_{2}(w)$ (for $x_{1}$ and $x_{2}$, respectively) are calculated according to (4). The asymmetry ratio, within the frequency range $w_{1}$ to $w_{2}$, can now be expressed as

$$
\Gamma_{\left(w_{1}, w_{2}\right)}=\frac{\left|\sum_{w_{1}}^{w_{2}} h_{1}^{2}(w)-\sum_{w_{1}}^{w_{2}} h_{2}^{2}(w)\right|}{\sum_{w_{1}}^{w_{2}} h_{1}^{2}(w)+\sum_{w_{1}}^{w_{2}} h_{2}^{2}(w)} .
$$

We shall now illustrate the calculation of asymmetry ratio on two signals containing sinusoids at two frequencies

$$
\begin{aligned}
f_{1} & =13 \mathrm{~Hz} \text { and } f_{2}=47 \mathrm{~Hz} \\
t & =1 / f s, \ldots, 2 s \text { and } f s=10 \mathrm{kHz} \\
x_{1} & =\cos \left(2 \pi f_{1} t\right)+\cos \left(2 \pi f_{2} t\right) \\
x_{2} & =1.7 \cos \left(2 \pi f_{1} t\right)+1.3 \cos \left(2 \pi f_{2} t\right) .
\end{aligned}
$$

Fig. 5 shows the asymmetry ratio with the lateralization evident at frequencies $f_{1}=13 \mathrm{~Hz}$ and $f_{2}=47 \mathrm{~Hz}$.

\section{COGNITIVE EXPERIMENTS}

To illustrate the advantages of using BEMD for estimating asymmetry, we considered its performance in two experimental

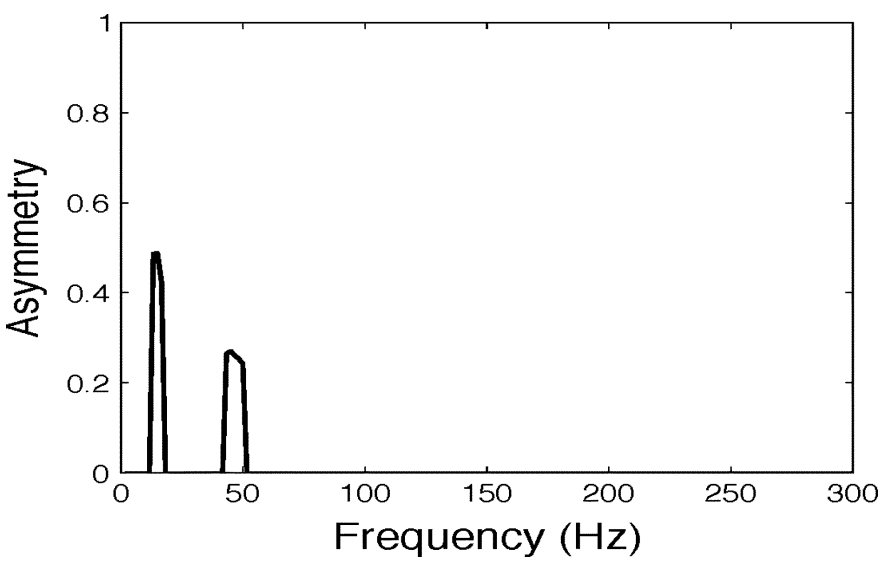

Fig. 5. Asymmetry ratio obtained using BEMD, for two channels. $x_{1}=\cos \left(2 \pi f_{1} t\right)+\cos \left(2 \pi f_{2} t\right)$ and $x_{2}=1.7 \cos \left(2 \pi f_{1} t\right)+1.3 \cos \left(2 \pi f_{2} t\right)$ with $f_{1}=13 \mathrm{~Hz}$ and $f_{2}=47 \mathrm{~Hz}$.

settings. In the first experiment, EEG was band-pass filtered to occupy a certain frequency band and the level of asymmetry is calculated between noisy realisations of the conditioned signal. In the second experiment, EEG asymmetry features are used to identify different mental tasks in a BCI application.

\section{A. Bandpass Filtered EEG}

1) Setting: EEG data with a sampling frequency of $250 \mathrm{~Hz}$ was obtained from electrode P3 according to the 10-20 system [25] and filtered using a Butterworth filter such that it occupied specific frequency bands. For an EEG signal conditioned to occupy a frequency region denoted by "band," $x_{\text {band }}$, the asymmetry ratio was calculated for the signal pair

$$
x_{\text {band }}+v_{1}, \quad x_{\text {band }}+v_{2}
$$

where $v_{1}$ and $v_{2}$ are different realisations of WGN. Thus, the expected asymmetry result should be zero in the frequency region denoted by band and nonzero within the remainder of the spectrum. The asymmetry ratios obtained using three different spectrum estimation techniques (periodogram $P_{x x}$ using Bartlett window, EMD and BEMD) were calculated for $x_{\text {band }}$ conditioned to occupy different frequency bands (delta, theta, alpha, beta and gamma bands) for four different levels of SNR $(5,0,-5,-10 \mathrm{~dB})$. Their performances were evaluated by the index $\left(\rho_{\text {band }}\right) /\left(\rho_{\text {spec }}\right) \times 100$, where $\rho_{\text {band }}$ is the total asymmetry detected in the correct frequency band, as dictated by the frequency range that $x_{\text {band }}$ is conditioned to occupy for a given simulation, and $\rho_{\text {spec }}$ is the total asymmetry detected in the full spectrum. The performance index for an accurate estimation of asymmetry will therefore give zero while poorer results will approach unity. The index was estimated and averaged over 50 independent trials for different realisations of $v_{1}$ and $v_{2}$.

2) Results: Fig. 6 shows the asymmetry ratio calculated for the signal pair $x_{\text {band }}+v_{1}, x_{\text {band }}+v_{2}$ at intervals of $0.125 \mathrm{~Hz}$ where $x_{\text {band }}$ was conditioned to occupy the alpha band and with SNR $0 \mathrm{~dB}$. As expected, all the algorithms showed lower asymmetry in the alpha band than in the other frequency regions. The BEMD asymmetry estimate was the most accurate of all the considered algorithms. 


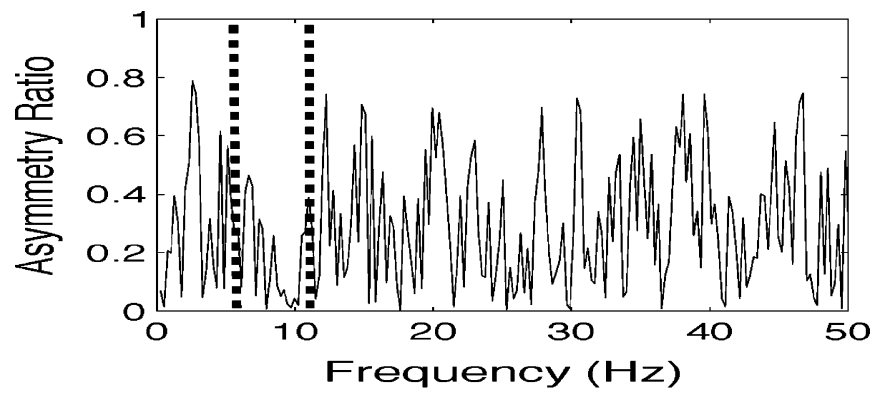

(a)

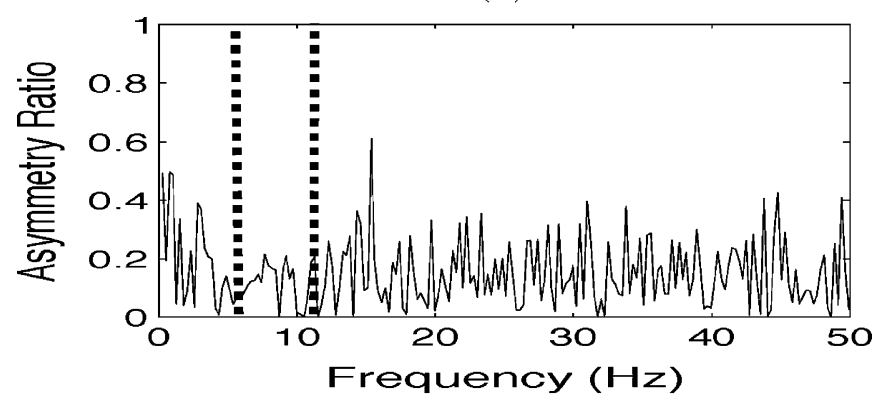

(b)

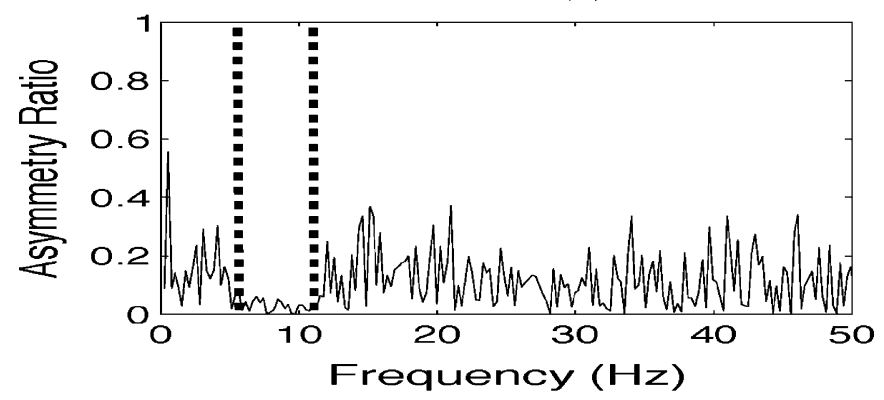

(c)

Fig. 6. Asymmetry graphs for the alpha band signals (between the dashed lines) in $0 \mathrm{~dB}$ WGN. These asymmetry ratios were computed at intervals of $0.125 \mathrm{~Hz}$. BEMD produced the lowest and most stable asymmetry in the alpha band. (a) Periodogram. (b) EMD. (c) BEMD.

Table IV shows a comparison of the performance for all the three algorithms, periodogram $P_{x x}$, EMD and BEMD, using the performance index $\left(\rho_{\text {band }}\right) /\left(\rho_{\text {spec }}\right) \times 100$ for all considered frequency bands and noise levels. In 16 of the 20 considered simulations, the performance of BEMD exceeds those of the periodogram and EMD. In the other four cases, the performance difference between BEMD and the superior algorithm was not significant. In several instances the periodogram outperformed the standard EMD algorithm, due to the linearity of the data, for which the periodogram is ideally suited. ${ }^{3}$

\section{B. BCI Application}

1) Setting: The EEG data was recorded by Keirn and Aunon who conducted several mental task experiments ${ }^{4}$. These tasks were specially chosen for their known hemispheric brainwave asymmetry [11].

\footnotetext{
${ }^{3}$ The linearisation of nonlinear data is a well-known consequence of linear filtering operations [26].

${ }^{4}$ Publicly available from http://www.cs.colostate.edu/eeg/index.html.
}

TABLE IV

Performance Evaluation, $\left(\rho_{\text {band }}\right) /\left(\rho_{\text {spec }}\right) \times 100$, FOR ASYMMETRY ESTIMATION BETWEEN SIGNALS $x_{1}$ AND $x_{2}$ AT DIFFERENT FREQUENCIES AND SNR

\begin{tabular}{|c|c|c|c|c|}
\hline \multirow{2}{*}{ Band } & Algorithm & $P_{x x}$ & EMD & BEMD \\
& SNR $_{\text {init. }}$ & & & \\
\hline \multirow{4}{*}{ Delta } & 5 & $1.4 \pm 0.6$ & $2.7 \pm 1.1$ & $\mathbf{1 . 3} \pm \mathbf{1 . 2}$ \\
& 0 & $1.4 \pm 0.8$ & $2.2 \pm 0.6$ & $\mathbf{1 . 3} \pm \mathbf{0 . 5}$ \\
& -5 & $1.9 \pm 0.8$ & $2.0 \pm 0.5$ & $\mathbf{1 . 3} \pm \mathbf{0 . 9}$ \\
& -10 & $\mathbf{1 . 6} \pm \mathbf{0 . 8}$ & $2.0 \pm 0.3$ & $1.7 \pm 0.4$ \\
\hline \multirow{3}{*}{ Theta } & 5 & $0.5 \pm 0.2$ & $1.4 \pm 0.3$ & $\mathbf{0 . 4} \pm \mathbf{0 . 1}$ \\
& 0 & $0.8 \pm 0.3$ & $1.6 \pm 0.2$ & $\mathbf{0 . 6} \pm \mathbf{0 . 2}$ \\
& -5 & $1.7 \pm 0.6$ & $1.7 \pm 0.3$ & $\mathbf{1 . 1} \pm \mathbf{0 . 3}$ \\
& -10 & $2.2 \pm 0.9$ & $\mathbf{1 . 6} \pm \mathbf{0 . 4}$ & $1.7 \pm 0.4$ \\
\hline \multirow{3}{*}{ Alpha } & 5 & $1.4 \pm 0.6$ & $2.0 \pm 0.4$ & $\mathbf{1 . 0} \pm \mathbf{0 . 3}$ \\
& 0 & $2.1 \pm 0.8$ & $1.9 \pm 0.3$ & $\mathbf{1 . 1} \pm \mathbf{0 . 3}$ \\
& -5 & $2.4 \pm 0.9$ & $1.8 \pm 0.3$ & $\mathbf{1 . 6} \pm \mathbf{0 . 5}$ \\
& -10 & $2.5 \pm 1.2$ & $2.2 \pm 0.3$ & $\mathbf{2 . 1} \pm \mathbf{0 . 5}$ \\
\hline \multirow{3}{*}{ Beta } & 5 & $5.1 \pm 1.1$ & $7.5 \pm 0.5$ & $\mathbf{3 . 4} \pm \mathbf{0 . 3}$ \\
& 0 & $7.5 \pm 1.7$ & $7.8 \pm 0.5$ & $\mathbf{5 . 1} \pm \mathbf{0 . 5}$ \\
& -5 & $9.9 \pm 2.0$ & $8.0 \pm 0.5$ & $\mathbf{5 . 2} \pm \mathbf{0 . 7}$ \\
& -10 & $11.8 \pm 2.6$ & $8.1 \pm 0.6$ & $\mathbf{7 . 9} \pm \mathbf{0 . 8}$ \\
\hline \multirow{3}{*}{ Gamma } & 5 & $\mathbf{1 . 4} \pm \mathbf{0 . 5}$ & $3.7 \pm 0.3$ & $1.6 \pm 0.2$ \\
& 0 & $\mathbf{2 . 4} \pm \mathbf{0 . 7}$ & $3.9 \pm 0.4$ & $2.6 \pm 0.3$ \\
& -5 & $4.0 \pm 1.2$ & $4.3 \pm 0.5$ & $\mathbf{3 . 4} \pm \mathbf{0 . 4}$ \\
& -10 & $5.3 \pm 1.6$ & $4.5 \pm 0.5$ & $\mathbf{4 . 4} \pm \mathbf{0 . 5}$ \\
\hline
\end{tabular}

- Task 1-Baseline Measurements (B): No mental task was performed in this experiment. The subject was told just to relax and think of nothing.

- Task 2-Complex Problem Solving (M): The subject was required to solve a nontrivial multiplication problem without vocalizing. The problems were nonrepeating and were designed so that an immediate answer was not apparent. The subject verified at the end of the task whether or not he had arrived at a solution and no subject completed the task before the end of the $10 \mathrm{~s}$ recording session.

- Task 3-Geometric Figure Rotation (R): The subject was given a drawing of a complex three dimensional block figure to study. After $30 \mathrm{~s}$, the figure was removed and the subject was instructed to visualize the object being rotated about an axis.

- Task 4-Mental Letter Composing (L): The subject composed a letter to a friend or relative without vocalizing.

- Task 5-Visual Counting (C): The subject was instructed to imagine a blackboard and to visualize numbers being written on the board. And the subject counted from the previous number and wrote it on the board after erasing the previous number.

Electrodes P3 and P4 were selected to calculate the asymmetry ratio between left and right hemisphere among all the recorded channels on the position $\mathrm{O} 1, \mathrm{O} 2, \mathrm{P} 3, \mathrm{P} 4, \mathrm{C} 3$, and $\mathrm{C} 4$ defined by the 10-20 system of electrode placement, at a $250 \mathrm{~Hz}$ data rate. The data were measured for $10 \mathrm{~s}$ during each task and each task was repeated five times per session. Recordings were made with reference to electrically linked mastoids A1 and A2. For more details about the experiments we refer to [11].

Asymmetry ratios in the delta, theta, alpha, beta and gamma bands, $\Gamma_{(1,3)}, \Gamma_{(4,7)}, \Gamma_{(8,13)}, \Gamma_{(14,30)}, \Gamma_{(36,44)}$, calculated using the periodogram $\left(P_{x x}\right)$, EMD and BEMD were used in a classifier. A support vector machine (SVM) [27] with Gaussian 
TABLE V

Classification Rates Obtained Using Periodogram (Shown as “ $P_{x x}$ ”), EMD AND BEMD Based on Asymmetry Estimation For Four SubJeCts AND Multiple Mental Task Combinations (Baseline Measurement (B), Complex Problem Solving (M), Geometric Figure Rotation (R), Mental

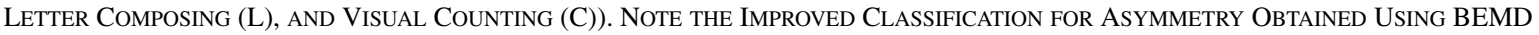

\begin{tabular}{|c|c|c|c|c|c|c|c|c|c|c|c|c|}
\hline & \multicolumn{3}{|c|}{ Subject 1} & \multicolumn{3}{|c|}{ Subject 2} & \multicolumn{3}{|c|}{ Subject 3} & \multicolumn{3}{|c|}{ Subject 4} \\
\hline & BEML & EMD & $P_{x x}$ & BEM & ËMD & $P_{x x}$ & BEMD & ËMD & $P_{x x}$ & BEMD & EMD & $P_{x x}$ \\
\hline$B, C$ & 86.3 & 77.5 & 79.5 & 83.3 & 45 & 71.8 & \begin{tabular}{|l|l|}
77.4 \\
\end{tabular} & 58.8 & 53.8 & 69 & 58.8 & \\
\hline$B, L$ & 52.5 & 63.8 & 49.5 & 58.1 & 47.5 & 63.8 & 50.8 & 37.5 & 53.6 & 56 & 55 & 53.8 \\
\hline$B, M$ & 51.3 & 28.8 & 38 & 51.3 & 60 & 59.4 & 85.7 & 62.5 & 71.8 & 85.5 & 67.5 & 83.5 \\
\hline$B, R$ & 95.5 & 78.8 & 70 & 51.3 & 45 & 46 & 50.8 & 70 & 48 & 55.4 & 45 & 54 \\
\hline$L, C$ & 70.6 & 70 & 63.1 & 51.3 & 61.3 & 46 & 88 & 77.5 & 72.5 & 64 & 53.8 & 51.8 \\
\hline$L, R$ & 64.5 & 68.8 & 62.5 & 65 & 70 & 61.9 & 47 & 73.5 & 44 & 55.8 & 46.3 & 44.3 \\
\hline$M, C$ & 85 & 71.8 & 81.8 & 75.8 & 42.5 & 43 & 58.5 & 55 & 56.2 & 88.5 & 88.5 & 68.8 \\
\hline$M, L$ & 49.5 & 48.8 & 43.5 & 72 & 45 & 51 & 74.8 & 56.3 & 76.5 & 81.25 & 58.8 & 60.3 \\
\hline$M, R$ & 96 & 83.75 & 74 & 78 & 63.8 & 40.3 & 70.5 & 63.8 & 57.2 & 58 & 73.8 & 55.5 \\
\hline$R, C$ & 57.8 & 42.5 & 50.3 & 87.5 & 46.3 & 71.3 & 81.25 & 66.3 & 59.75 & 81 & 72.5 & 61.5 \\
\hline Average & 70.9 & 63.8 & 61.2 & 67.3 & 52.6 & 55.5 & 68.5 & 62.1 & 59.3 & 69.5 & 62 & 9.3 \\
\hline & 96 & 83.8 & 8.8 & 87.5 & 63.8 & 71.25 & 88 & 77.5 & 76.5 & 88.5 & 88.5 & 33.5 \\
\hline Best & $\mathrm{M}, \mathrm{R}$ & M,R & $\mathrm{M}, \mathrm{C}$ & $\mathrm{R}, \mathrm{C}$ & $\mathrm{M}, \mathrm{R}$ & $\mathrm{B}, \mathrm{C}$ & $\mathrm{L}, \mathrm{C}$ & $\mathrm{L}, \mathrm{C}$ & M,L & M.C & $\mathrm{M}, \mathrm{C}$ & $\mathrm{B}, \mathrm{M}$ \\
\hline
\end{tabular}

kernel, (the code obtained from [28]), was used to classify between two different mental tasks. The different combinations of two mental tasks are shown in Table V. Each combination had 20 samples of $4 \mathrm{~s}$ segment data because in the original recording [11] each subject had five trials for a task and the length of each EEG data was $8 \mathrm{~s}$ long discarding the first and last $1 \mathrm{~s}$ concerned about noise. The numbers of training sets and test sets were respectively 12 and 8 . The classification was repeated 50 times while mixing the sample order, and the final classification result was the average of these outcomes.

2) Results: Table V shows the classification performances for the four subjects using the BEMD, EMD and periodogram. Of the 40 considered simulation scenarios, EMD and BEMD asymmetry outperformed periodogram asymmetry for 36 . On average, the BEMD-based asymmetry calculation had a classification accuracy of $70 \%$, a $10 \%$ improvement over periodogram-based asymmetry and a 7\% improvement over EMD-based asymmetry.

These results fully illustrate the advantages of empirical mode decomposition and its algorithms for asymmetry estimation of real-world data. In simulations presented in previous sections of this paper, the signals of interest were linear: band-pass filtered EEG data. Algorithms based on Fourier theory such as the Periodogram are, by design, suitable for linear signal statistics and its performance exceeded that of standard EMD, and BEMD to a lesser extent, in some instances. However, for fully nonlinear and nonstationary data sources, the performance of both EMD and BEMD facilitated an improvement over traditional linear asymmetry estimates.

In practice, asymmetry features are often combined with spectral power features to achieve separation between mental tasks [29]. As our aim was to address only asymmetry performance, it is shown that BEMD asymmetry achieved classification accuracy exceeding $85 \%$ in some instances.

Recently, empirical mode decomposition has been extended to multivariate EMD by Rehman et al. [30] in order to make EMD suitable for multichannel signal processing. It was also illustrated [31] how the noise assisted MEMD allows for enhanced localization in time-frequency analysis. In future work, multivariate EMD techniques will be utilized to extract more accurate and robust asymmetry features.

\section{CONCLUSION}

It has been shown that complex extension of the EMD algorithm provides a robust estimate of asymmetry for nonlinear and nonstationary data. One such extension, bivariate empirical mode decomposition facilitates enhanced multicomponent analysis and improved marginal Hilbert spectrum estimation, critical to the calculation of asymmetry. A statistical assessment of the proposed framework has been presented for both synthetic signals and for EEG from a BCI application. The BEMD-based asymmetry results are shown to be highly localized in frequency and robust to additive noise.

\section{REFERENCES}

[1] A. Akrami, S. Solhjoo, A. Motie-Nasrabadi, and M. Hashemi-Golpayegani, "EEG-based mental task classification: Linear and nonlinear classification of movement imagery," in Proc. 2005 IEEE Eng. Med. Biol. 27th Annu. Conf., 2005, pp. 4626-4629.

[2] J. Knott and T. Tjossem, "Bilateral encephalograms from normal speakers and stutterers," J. Exp. Psychol., vol. 32, no. 4, pp. 357-362, 1943.

[3] J. Knott and L. Travis, "Bilaterally recorded brain potentials from normal speakers and stutterers," J. Speech Disorders, vol. 2, pp. 239-241, 1937.

[4] D. Galin and R. Ornstein, "Lateral specialization of cognitive mode: An EEG study," Psychophysiology, vol. 9, no. 4, pp. 412-418, 1972.

[5] R. J. Davidson, J. P. Chapman, L. J. Chapman, and J. B. Henriques, "Asymmetrical brain electrical activity discriminates between psychometrically-matched verbal and spatial cognitive tasks," Psychophysiology, vol. 27, no. 5, pp. 528-543, 1990.

[6] M. J. Hoptman and R. J. Davidson, "Baseline EEG asymmetries and performance on neuropsychological tasks," Neuropsychologia, vol. 36, no. 12 , pp. 1343-1353, 1998.

[7] S. A. Weems, E. Zaidel, S. Berman, and M. A. Mandelkern, "Asymmetry in alpha power predicts accuracy of hemispheric lexical decision," Clin. Neurophysiol., vol. 115, no. 7, pp. 1575-1582, 2004.

[8] G. McKee, B. Humphrey, and D. W. McAdam, "Scaled lateralization of alpha activity during linguistic and musical tasks," Psychophysiology, vol. 10, no. 4, pp. 441-443, 1973.

[9] E. Donchin, M. Kutas, and G. McCarthy, , S. Harnad, R. D. L. Goldstein, J. Jaynes, and G. Krauthamer, Eds., Lateralization in the Nervous System. New York: Academic, 1977.

[10] R. Palaniappan, "Utilizing gamma band to improve mental task based brain-computer interface design," IEEE Trans. Neural Syst. Rehabil. Eng., vol. 14, no. 3, pp. 299-303, Sep. 2006.

[11] Z. A. Keirn and J. I. Aunon, "A new mode of communication between man and his surroundings," IEEE Trans. Biomed. Eng., vol. 37, no. 12, pp. 1209-1214, Dec. 1990.

[12] M. Putten and D. Tavy, "Continuous quantitative EEG monitoring in hemispheric stroke patients using the brain symmetry index," Stroke, vol. 35, pp. 2489-2492, 2004. 
[13] R. J. Davidson and K. Hugdahl, "Baseline asymmetries in brain electrical activity predict dichotic listening performance," Neuropsychology, vol. 10, no. 2, pp. 241-246, 1996.

[14] N. E. Huang, Z. Shen, S. R. Long, M. L. Wu, H. H. Shih, Z. Quanan, N. C. Yen, C. C. Tung, and H. H. Liu, "The empirical mode decomposition and the Hilbert spectrum for nonlinear and non-stationary time series analysis," in Proc. R. Soc. A, 1998, vol. 454, pp. 903-995, 1971 Ed..

[15] C. M. Sweeney-Reed and S. J. Nasuto, "A novel approach to the detection of synchronisation in EEG based on empirical mode decomposition," J. Computat. Neurosci., vol. 23, no. 1, pp. 79-111, 2007.

[16] D. Looney, L. Li, T. Rutkowski, D. P. Mandic, and A. Cichocki, "Ocular artifacts removal from EEG using EMD," in Proc. 1st Int. Conf. Cognitive Neurodynamics, 2007, pp. 831-835.

[17] D. Looney and D. P. Mandic, "Multi-scale image fusion using complex extensions of EMD," IEEE Trans. Signal Process., vol. 57, no. 4, pp. 1626-1630, Apr. 2009.

[18] G. Rilling, P. Flandrin, and P. Gongalves, "On empirical mode decomposition and its algorithms," in Proc. IEEE-EURASIP Workshop Nonlinear Signal Image Process., 2003.

[19] L. Cohen, "Instantaneous anything," in Proc. IEEE Int. Conf. Acoust., Speech Signal Process. (ICASSP), 1993, vol. 5, pp. 105-108.

[20] X. Li, Z. Liang, L. Voss, and J. Sleigh, "Analysis of depth of anesthesia with Hilbert-Huang spectral entropy," Clin. Neurophysiol., vol. 119, no. 11, pp. 2465-2475, 2008.

[21] X. Li, "Temporal structure of neuronal population oscillations with empirical model decomposition," Phys. Lett. A, vol. 356, pp. 237-241, 2006.

[22] D. Chen, D. Li, M. Xiong, H. Bao, and X. Li, "GPGPU-aided ensemble empirical-mode decomposition for EEG analysis during anesthesia," IEEE Trans. Inf. Technol. Biomed., vol. 14, no. 6, pp. 1417-1427, Nov. 2010.

[23] G. Rilling, P. Flandrin, P. Gongalves, and J. M. Lilly, "Bivariate empirical mode decomposition," IEEE Signal Process. Lett., vol. 14, no. 12, pp. 1-4, Dec. 2007.

[24] B. Weng and K. E. Barner, "Optimal and bidirectional optimal empirical mode decomposition," in Proc. IEEE Int. Conf. Acoustics, Speech, Signal Process. (ICASSP'07), Apr. 2007, vol. 3, pp. 1501-1504.

[25] H. H. Jasper, "The ten twenty electrode system of the international federation," Electroencephalogr. Clin. Neurophysiol., vol. 10, pp. 371-375, 1958.

[26] M. Chen, T. Gautama, and D. P. Mandic, "An assessment of qualitative performance of machine learning architectures: Modular feedback networks," IEEE Trans. Neural Networks, vol. 19, no. 1, pp. 183-189, Jan. 2008.

[27] H. Drucker, C. J. Burges, L. Kaufman, A. Smola, and V. Vapnik, Support Vector Regression Machines. Cambridge, MA: MIT Press, 1996.

[28] S. Canu, Y. Grandvalet, V. Guigue, and A. Rakotomamonjy, "SVM and kernel methods matlab toolbox," in Perception Systems et Information, INSA de Rouen, Rouen, France, 2005.

[29] M. Golz, D. Sommer, M. Chen, D. Mandic, and U. Trutschel, "Feature fusion for the detection of microsleep events," J. VLSI Signal Process., vol. 49, pp. 329-342, 2007.

[30] N. Rehman and D. P. Mandic, "Multivariate empirical mode decomposition," in Proc. R. Soc., 2010, vol. 466, pp. 1291-1302.

[31] N. Rehman and D. P. Mandic, "Filter bank property of multivariate empirical mode decomposition," IEEEE Trans. Signal Process., 2011, accepted for publication.

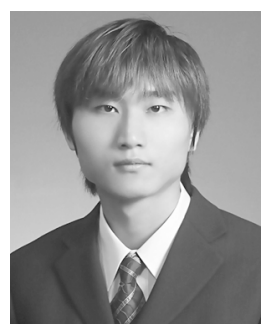

Cheolsoo Park received the B.Eng. degree (cum laude honors) in electrical engineering from Sogang University, Seoul, South Korea, in 2004, and the M.Sc. degree in biomedical department from Seoul National University, Seoul, South Korea, in 2006. $\mathrm{He}$ is currently working toward the Ph.D. degree in adaptive nonlinear signal processing at Imperial College London, London, U.K.

His research interests are mainly in the area of biomedical signal processing, brain computer interface, and time-frequency analysis.

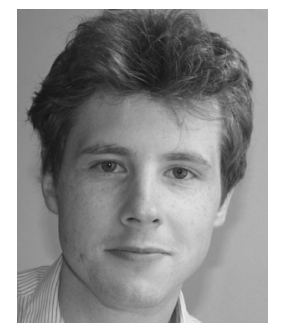

David Looney received the B.Eng. degree in electronic engineering from University College Dublin, Ireland, and the Ph.D. degree in adaptive data fusion from Imperial College, London, U.K., in 2011.

His research interests are mainly in the areas of data fusion, time-frequency analysis and matrix factorization.

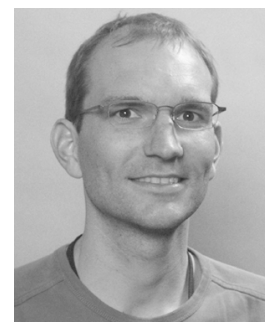

Preben Kidmose received the B.Sc. degree in electrical engineering from Engineering College Snderborg Teknikum, Denmark, the M.Sc. degree in engineering from Technical University of Denmark, and the $\mathrm{Ph}$.D. degree in signal processing from Technical University of Denmark

$\mathrm{He}$ is currently Professor (Docent) in Medical Technology at the Aarhus School of Engineering/Engineering College of Aarhus, Denmark. His area of interest include signal processing in hearing aids and medical devices, audio signal processing, machine learning, medical instrumentation, and biomedical system design.

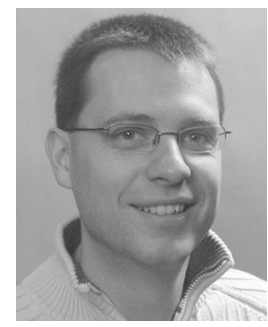

Michael Ungstrup received the M.Sc. degree in electrical engineering from the Technical University of Denmark.

He is currently employed by Widex A/S, Denmark. His areas of interest include signal processing for audio applications and communication.

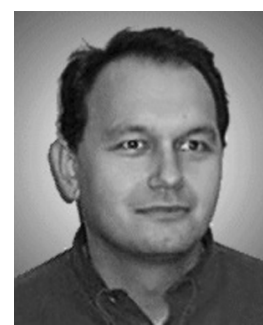

Danilo P. Mandic (SM'03) is a Reader in Signal Processing at Imperial College London. He has been working in the area of nonlinear adaptive signal processing and nonlinear dynamics. His publication record includes two research monographs titled Recurrent Neural Networks for Prediction (1st ed., 2001) and Complex Valued Nonlinear Adaptive Filters: Noncircularity, Widely Linear and Neural Models (1st ed., Wiley, 2009), an edited book titled Signal Processing for Information Fusion (Springer, 2008 ) and more than 200 publications on signal and image processing. He has been a Guest Professor at K.U. Leuven Belgium, TUAT Tokyo, Japan, and Westminster University, U.K., and a Frontier Researcher in RIKEN Japan. He has produced award winning papers and products resulting from his collaboration with industry. He has been an Associate Editor for the International Journal of Mathematical Modelling and Algorithms. 